\title{
Carnosine in exercise and disease: introduction to the International Congress held at Ghent University, Belgium, July 2011
}

\author{
Wim Derave $\cdot$ Craig Sale
}

Received: 13 March 2012/ Accepted: 27 March 2012/Published online: 22 April 2012

(C) Springer-Verlag 2012

In the historical city center of Ghent in Belgium, an international group of scientists gathered for a 3-day meeting dedicated to carnosine research. The meeting took place in July 2011, which is more than 10 years following the previous congress of this kind, organized by Alexander Boldyrev in Moscow in the year 2000. The Moscow meeting was held on the occasion of the centenary of Gulewitch and Amiradzhibi's discovery of the dipeptide carnosine in meat extract. A special issue with contributions from the invited participants was published in Biochemistry (Moscow) in 2000, entitled: "Biological role of carnosine in the functioning of excitable tissues" (Guest editor: A. A. Boldyrev; Original Russian text). The Ghent meeting was therefore considered the Second International Meeting on Carnosine. The souvenir medals that were created to mark the occasion of both meetings are displayed in Fig. 1.

A thorough understanding of the biological role of carnosine and its methylated analogues anserine and ophidine/ balenine, is still hampered by the relatively low frequency of scientific discoveries on this topic. However, in the 11-year period separating both meetings there has been an exponential increase in the number of publications and

This is the introduction paper to the Special Issue of Amino Acids, entitled 'Carnosine in Exercise and Disease' and edited by Craig Sale and Wim Derave.

W. Derave $(\bowtie)$

Department of Movement and Sports Sciences, Ghent

University, Watersportlaan 2, 9000 Ghent, Belgium

e-mail: wim.derave@ugent.be

C. Sale

Biomedical, Life and Health Sciences Research Centre, School of Science and Technology, Nottingham Trent University, Nottingham, UK citations on carnosine (see Fig. 2). Indeed, significant progress was made in the fields of both biochemistry and medicine, as well as in sport science. The genes for carnosine synthase and carnosinase, the principal enzymes responsible for carnosine synthesis and degradation, were molecularly identified and cloned in 2003 and 2010, respectively. A polymorphism in the gene for the carnosine-degrading enzyme carnosinase was defined as an important genetic risk factor for diabetic nephropathy. The evidence for a therapeutic role of carnosine in aging and neurologic diseases has expanded and new target diseases, such as diabetes/metabolic syndrome, liver and kidney disease and cancer, have been successfully explored in animal experiments. Carnosinase-resistant analogues of carnosine are developed in the pharmaceutical industry for increased therapeutic potential and stability. Finally, the content of muscle carnosine appears to influence athletic performance and can be augmented by oral beta-alanine supplementation, making beta-alanine increasingly popular in the athletic community. Thus, a new meeting was timely and needed. The current special issue in Amino Acids provides a selection of contributions presented at the Ghent meeting, containing original papers, review articles and a new perspective.

Carnosine was originally discovered in skeletal muscle, where it has a far greater concentration compared to all other tissues. The regulation of the carnosine content in muscle is still incompletely understood, but the current knowledge is elegantly summarized in this special issue by Harris et al. (2012, this issue). A recent development of a non-invasive magnetic resonance spectroscopy (MRS) based method to quantify muscle carnosine in humans has further facilitated the research on this topic. Accordingly, a study based on over 260 male and female healthy humans aged 9-83 years (Baguet et al. 2012, this issue) indicates 

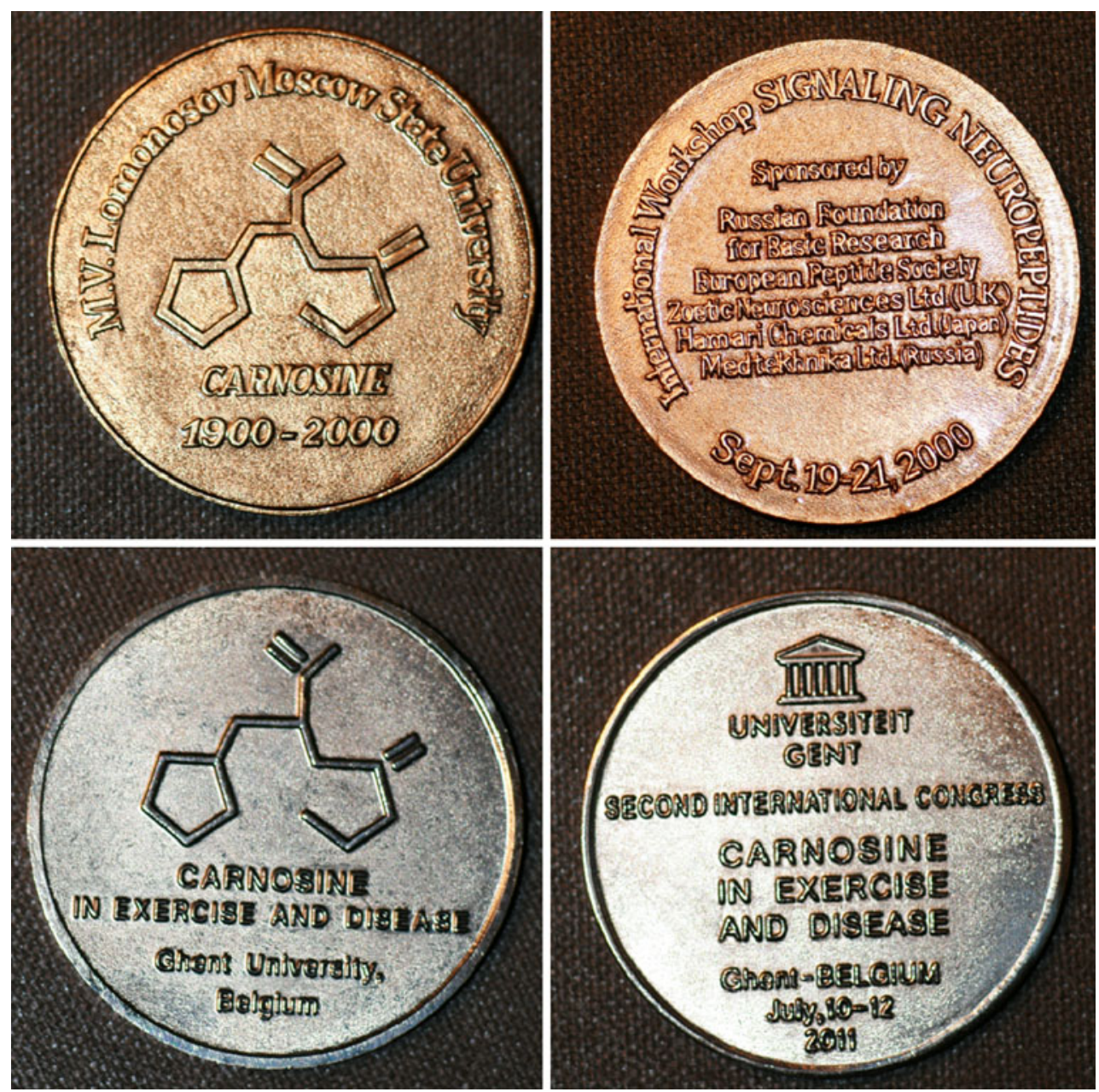

Fig. 1 Pictures of the medals commemorating the first (M.V. Lomonosov Moscow State University, Moscow, Russia; September 19-21,2000; upper panel) and the second (Ghent University, Ghent, Belgium; July 10-12, 2011; lower panel) International Congress on

Carnosine. New congresses in this series are planned and will be announced on the website of the Carnosine Consortium (http://users. unimi.it/carnosine_co/)

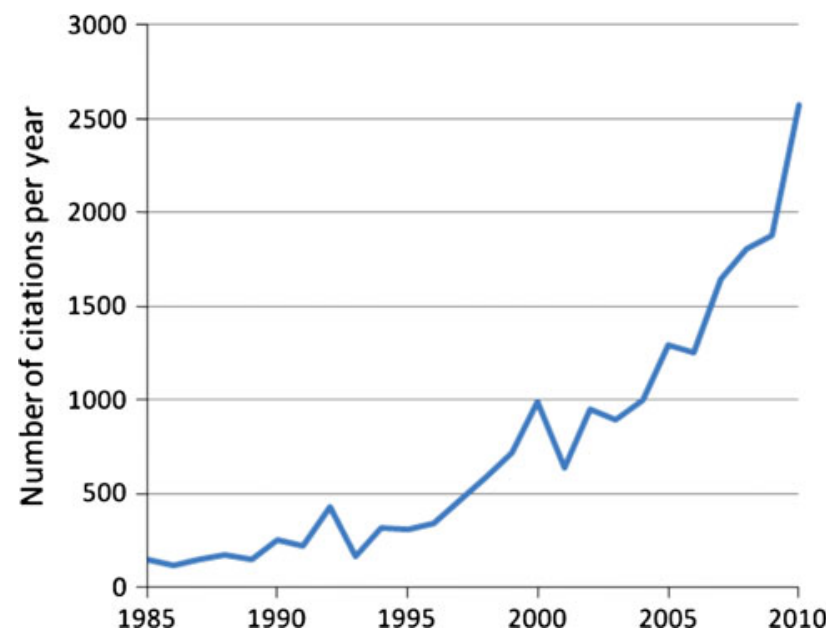

that gender differences in muscle carnosine develop during puberty and remain present until old age. A second MRSbased cross-sectional study indicates that muscle carnosine content is reduced in type-2 diabetic patients (Gualano et al. 2012, this issue), which can have important implications for the presumed therapeutic potential of carnosine in this disease.

Beta-alanine supplementation became a major contemporary topic in muscle carnosine research since Harris and co-workers showed in 2006 that several weeks of oral beta-alanine supplementation can elevate human muscle carnosine content by roughly $50 \%$. Many researchers subsequently explored whether muscle carnosine loading leads to enhanced performance capacity in high-intensity exercise. Since the first of these studies in 2007 , more than 15 manuscripts have been published on the ergogenic potential of beta-alanine and these are evaluated in this special issue through a meta-analysis (Hobson et al. 2012,

Graph of the yearly number of citations of Web of Scic publications on 'carnosine'. Note the exponential rise in citations in the last decade, leading to a current 10 -fold higher number compared to the early nineties 
this issue). This analysis reveals that a positive ergogenic effect is mainly observed in exhaustive exercise tests lasting 1-4 min. Repeated sprint performance in an exercise protocol designed to replicate games play, was not influenced by beta-alanine supplementation according to Saunders et al. (2012, this issue). New applications of betaalanine's effects on muscle function beyond the sport setting are now being explored. In this issue, Del Favero et al. (2012, this issue) show that in healthy Brazilians aged 60-80 years, beta-alanine can potently elevate muscle carnosine content $(+85 \%)$ as well as exercise capacity. This opens opportunities for the application of beta-alanine supplementation in the field of sarcopenia.

Given the expanding applications of beta-alanine, further development of the optimal dosing and delivery in relation to muscle carnosine loading is required. Stellingwerff et al. (2012, this issue) extensively review the properties and new insights into oral beta-alanine supplementation. A new development relates to the avoidance of unpleasant sensory symptoms (paraesthesia) when beta-alanine is ingested in doses larger than $800 \mathrm{mg}$, by supplementing with slow-release tablets. This strategy leads to slower absorption kinetics, improved whole body retention and reduced paresthesia (Decombaz et al. 2012, this issue).

The ergogenic effects of beta-alanine during exercise are traditionally ascribed to the $\mathrm{pH}$ buffering effects of carnosine in muscle, which was the first ever described biological role of carnosine. However, other mechanisms may equally contribute to the potentiating effects of elevated carnosine stores on the muscular force-generating properties such as the calcium release from the sarcoplasmic reticulum and the sensitivity of the contractile apparatus for calcium. Also the antioxidant properties of carnosine could be relevant to muscle function, as reactive oxygen species have been implicated in the development of fatigue. However, the study by Smith et al. (2012, this issue) showed only minimal effects of betaalanine supplementation on markers of oxidative stress in exercising women.

The current issue contributes to two main theories on the biological roles of carnosine in the brain. According to the first, carnosine functions as a protector of neuronal cells against oxidative stress. Kulebyakin et al. (2012, this issue) confirm that carnosine can suppress the activation of intracellular signaling cascades involved in excitotoxicity and cellular death. The second mechanism considers carnosine as a histidine reservoir and a histamine donor, thereby influencing neurotransmission in certain hypothalamic nuclei, such as the circadian clock. Nagai et al. review this topic and summarize the emerging role of carnosine in the autonomic nervous control of glycaemia, blood pressure, thermogenesis and lipolysis (Nagai et al. 2012, this issue).

Various biochemical properties of carnosine give the molecule the ability to inhibit the formation of cytotoxic products such as advanced glycation and lipoxidation end products (AGEs and ALEs), the mechanisms of which are reviewed by Vistoli et al. (2012, this issue). As these and other damaging compounds are involved in various pathological events, it can be expected that the potential therapeutic applications of carnosine and its analogues will continue to expand in the coming years. A paper by Ansurudeen et al. (2012, this issue) explores the role of carnosine and anserine in diabetic wound healing and Gaunitz et al. (2012, this issue) present a perspective on the promising preliminary investigations relating to the role of carnosine in the protection against tumor growth.

A general limitation to the therapeutic potential of carnosine is the high activity of human serum carnosinase. In contrast to most other mammals, humans rapidly hydrolyze carnosine once it appears in the circulation. This issue includes a paper in which the development of a new ELISA-based method is described to capture content and different conformational forms of carnosinase in human serum (Adelmann et al. 2012, this issue). Due to the action of serum carnosinase in humans, administered carnosine rapidly loses many of its protective properties, making it difficult to translate the numerous protective and therapeutic effects from rodents to human patients. The development of carnosinase-resistant carnosine analogues is therefore an intensive field of study and a major hurdle to be taken for future pharmaceutical progress. The papers by Vistoli et al. and Bellia et al. (2012, this issue) provide an excellent overview of the recent advances in the development of carnosine derivatives. Stvolinsky et al. (2012, this issue) have tested trolox derivatives of carnosine in the protection of animals against oxidative stress.

From the various contributions to the congress and this special issue, it can be concluded that carnosine is a unique and promising molecule. However, despite over 110 years of research, its biological roles are not fully understood at present. The potential medical applications are numerous but we must acknowledge that only very few of these have successfully entered the first phases of clinical trials. The number of patents on carnosinase-resistant carnosine derivatives is rapidly growing, which will hopefully lead to significant pharmaceutical advances in the near future. In contrast to the limited number of readily available medical applications, the use of carnosine loading by beta-alanine supplementation in sport science and the athletic community has made a successful jump to the market in the past 5 years. We hope that this dedicated journal issue 
can stimulate further scientific interest in this intriguing molecule.

Acknowledgment We would like to dedicate this Special Issue to the memories of Prof. Alexander Boldyrev (Moscow State University) and Dr. John Wise (Natural Alternatives International) who both passed away between the Congress in July and the completion of this Special Issue. The valuable contribution of Prof Boldyrev, Moscow State University, Russia, to this research field is unquestioned, whilst
Dr Wise of NAI, San Marcos, California, USA, provided a valuable contribution in promoting research in the area of muscle carnosine, its metabolism, the role of diet and its importance to exercise performance. He was also critical in re-awakening the interest of the role of this peptide in muscle.

Conflict of interest The authors declare that they have no conflict of interest. 